\title{
An electromagnetic system for planetary geophysical exploration
}

\author{
Roberto Marcialis $\left({ }^{1}\right)$, Sergio Pagnan $\left({ }^{1}\right)$, Giuseppe Bucci $\left({ }^{2}\right)$ and Cinthya Ottonello $\left({ }^{2}\right)$ \\ ${ }^{1}$ ) Istituto per l'Automazione Navale, Consiglio Nazionale delle Ricerche, Genova, Italy \\ (2) Dipartimento di Ingegneria Biofisica ed Elettronica, Università di Genova, Italy
}

\begin{abstract}
The paper presents a study, carried out in the context of TESEO project (Sistema di prospezione TDEM per l'ESplorazione gEOfisica dei pianeti), for the design of a TDEM (Time Domain Electromagnetic Method) system suitable for planetary subsurface exploration. The TDEM technique has been selected since it is based on the parameter of electrical conductivity, which is generally capable of discriminating geological layered structures in a fully non-invasive manner, down to depths ranging several hundred meters. Furthermore, TDEM does not need any material sample and intrinsically satisfies the planetary protection and cross contamination requirements. Finally, since the TDEM is a time-domain technique, it is in principle possible to retrieve information from different depths with a single measurement. This last peculiarity makes the TDEM technique much more expeditious and flexible than analogous techniques operating in the frequency domain. The paper describes the purposes of TESEO project, the requirements due to space application and the preliminary system design.
\end{abstract}

Key words TDEM - planetary exploration subsurface sounding - signal processing

\section{Introduction}

The surface of the bodies of the Solar System has been investigated by several missions and others are planned. Currently, Mars Global Surveyor is complementing the global map of Mars, producing high-resolution images of a small part of the planet by orbital missions. Other missions provided detailed images of the Jovian bodies, Venus, and Mercury.

These investigations, initially based upon optical and remote sensors, are becoming much less «remote» with the landing of robots on the surface of Solar System's bodies (Mars'

Mailing address: Dr. Roberto Marcialis, Istituto per l'Automazione Navale, Consiglio Nazionale delle Ricerche, Via De Marini 6, 16149 Genova, Italy; e-mail: roberto@ ian.ge.cnr.it
Pathfinder type) and include a wider range of parameters and measurements. In the next exploration programs, semi-autonomous roving vehicles capable of conducting science experiments on the surface of the planets are expected to be applied.

The Italian Space Agency (ASI) has also been engaged for several years in projects concerning in-situ planetary exploration. In the last call of the Scientific Research Program (July 2000), ASI recommended the submission of proposals focused on:

- Remote sensing of Mars surface, to be employed for orbital missions.

- Geophysical sounding of Mars soil and subsurface, for in-situ missions.

- Radar sounding of Jovis satellite, Europa.

In particular, drilling systems for subsurface analysis and sample collection as well as instrumentation suitable for installation aboard rovers were recommended for Mars exploration.

In response to this call, the TESEO project (Sistema di prospezione TDEM per l'ESplo- 
razione $\mathrm{gEO}$ fisica dei pianeti) has been conceived and submitted to Italian Space Agency's review for the realization of an electromagnetic (EM) system for geological prospecting of subsurface structure of the planetary bodies.

The project brings together the scientific and technological knowledge of experts in the fields of geophysical prospecting, planetary exploration research and EM signal processing.

According to the expertise of the authors, the paper presents the main guidelines of TESEO project, mainly focussing on the engineering and telecommunication aspects of the research.

Section 2 describes the purposes of the project and problems to be faced when application of consolidated technologies is transferred from ground to space application.

The approach followed in order to design a system adapted to work in extreme environmental conditions and with reduced data collection and processing resources is presented in Section 3. This section deals with a case study: the design of a TDEM (Time-Domain Electromagnetic Measurements) system to be installed on board DEEDRI, the Italian Drill developed for collecting samples of Mars subsurface (Mars Sample Return Lander). In the section, the solutions adopted for signal acquisition and processing are particularly stressed. Section 4 draws some conclusions and future prospective, addressing the impact that the research may have on systems used for Earth's sounding.

\section{Objectives of the TESEO project}

The abundance of data from planets' surfaces collected in past missions has provided a remarkable background for geological analysis. However, several of the questions which arose should be addressed to the investigation of the subsurface.

TESEO represents a possible solution to the request of performing non intrusive in-situ geophysical measures, which are of immediate interest for Mars.

Several reasons induce TDEM to be chosen for the development of a system for EM measurements, among the many geophysical sound- ing techniques (Nabighian, 1984, 1993). TDEM is based on the parameter of electrical conductivity, which is generally able to discriminate geological layered structures. Since it is a time-domain technique (recording of a relaxation transient following a proper excitation), it is in principle possible to retrieve information from different depths with a single measurement, speeding up the execution of highdensity surveys over large soil areas. This last peculiarity makes the TDEM technique much more expeditious and flexible than analogous techniques operating in the frequency domain. It is a low-cost and light-weight technology. Moreover, the resistivity of a rock can undergo notable variations due to water presence. Therefore, it may result very useful in detecting water/ice signatures. Unlike other techniques, TDEM does not need material samples and intrinsically satisfies the Planetary Protection and Cross Contamination requirements.

TESEO system will be designed in order to achieve the following main objectives:

- Measuring electrical conductivity profiles of planet subsoil layers.

- Identifying ground water/ice signatures.

- Performing passive measurements of EM environment.

The above-mentioned objectives are perhaps attainable from a TDEM system operating on the Earth, but would be much more complex if these techniques had to be accomplished from space. There are several constraints that cannot be disregarded:

- Mass and power budget limitations.

- Possibility of transmitting to the ground only a reduced amount of collected data.

- Possibility of scanning a limited area of the planet's surface by TDEM.

- EM interferences with other science experiments.

- Hostile environmental working conditions.

The aforementioned limitations may compromise inspection depth, measure quality and data interpretation. Improvements are obtainable by adopting ad hoc mechanical and electronic solutions. In particular, as concerns the acquisition system and measure allocation, the design will be focused on the following aspects: choice of hardware components operating in very 
critical conditions, design of efficient data preprocessing procedures, design of data compression procedures.

It is also necessary, during the early phases of TESEO development, to understand the type, quality and interpretation possibility of the data that will be obtained by the instrument. The geophysical partners involved in the project will simulate the geological setting of the investigation targets and will model the possible data obtained in the selected geological settings, in order to suggest a possible output of the EM sounding.

Accordingly, a set of experimental tests will be planned on the Earth in representative geological areas for planetary application. Such measures will be used for instrument validation as well as performance evaluation versus stringent restrictions imposed by space application. In particular, the main tasks of the project consist of:
1) Design and implementation of the measuring equipment.

2) Design and implementation of the hardware and software systems for data acquisition, processing and ground reconstruction.

3) Experimental surveys for Earth-based tests and instrument validation.

4) Processing and interpretation of the experimental data sets.

5) System specifications for space application and evaluation of missions of interest for TESEO installation.

\section{A case study: the TDEM system designed for DEEDRI Payload}

In order to give an idea of the problems to be faced when operating in space, in this section the case study of a TDEM system,

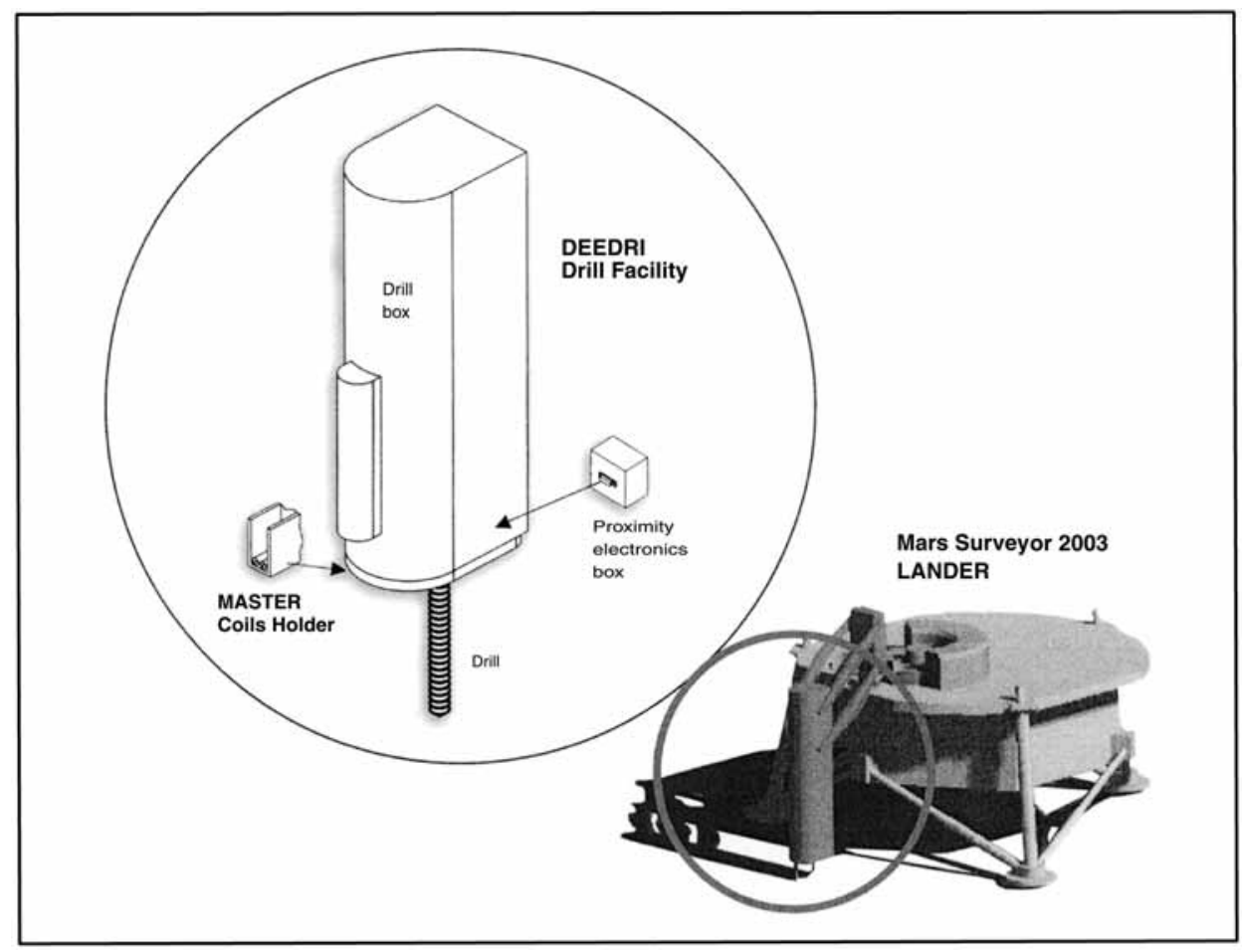

Fig. 1. Allocation of the TDEM instrument on board of DEEDRI. 
named MASTER (MArs subsurface Sounding by Time-domain Electromagnetic measuRements), is presented (Tacconi et al., 2000).

MASTER has been designed for allocation aboard DEEDRI, the Italian Drill developed for collecting samples of Mars subsurface. DEEDRI represents one of the ten Additional Payloads that are scheduled to fly aboard the mission Mars Sample Return (fig. 1).

MASTER is based on the TDEM technique. The basic configuration of a TDEM instrument consists of the so-called central loop induction coil, which is used to carry out time-domain EM soundings. The receiver, a sensitive magnetometer, may be placed concentric with the source loop or may be a separated loop.

Ground application systems generally use transmitting coils from $1 \mathrm{~m}$ up to some tens of meters wide, driven by currents from one Ampere up to tens of Amperes, depending on the required inspection depth.

However, the installation aboard DEEDRI imposes some specific requirements, such as:

- Minimizing the total mass of the system (total weight less than $1000 \mathrm{~g}$ ).
- Using components working in a hostile environment (e.g., temperature range $[-130,85]^{\circ} \mathrm{C}$ ).

- Using very low power consumption components (available average power of the order of some watts).

- Reducing data memory allocation and computation time.

- Limiting ground transmission resources (transmission channel capacity less than 50 Kbyte per Martian Day).

According to these specifications, the system presented in Section 3.1 was designed. In addition, in order to overcome drawbacks due to mechanical and electrical design limitations, which may compromise the obtainable data, acquisition and processing procedures for critical Signal-to-Noise Ratio (SNR) measure treatment were implemented. Sections 3.2, 3.3 and 3.4 present the followed approach and some simulation results.

\subsection{MASTER instrument configuration}

The instrument developed for the DEEDRI uses a set of two equal concentric coils (trans-

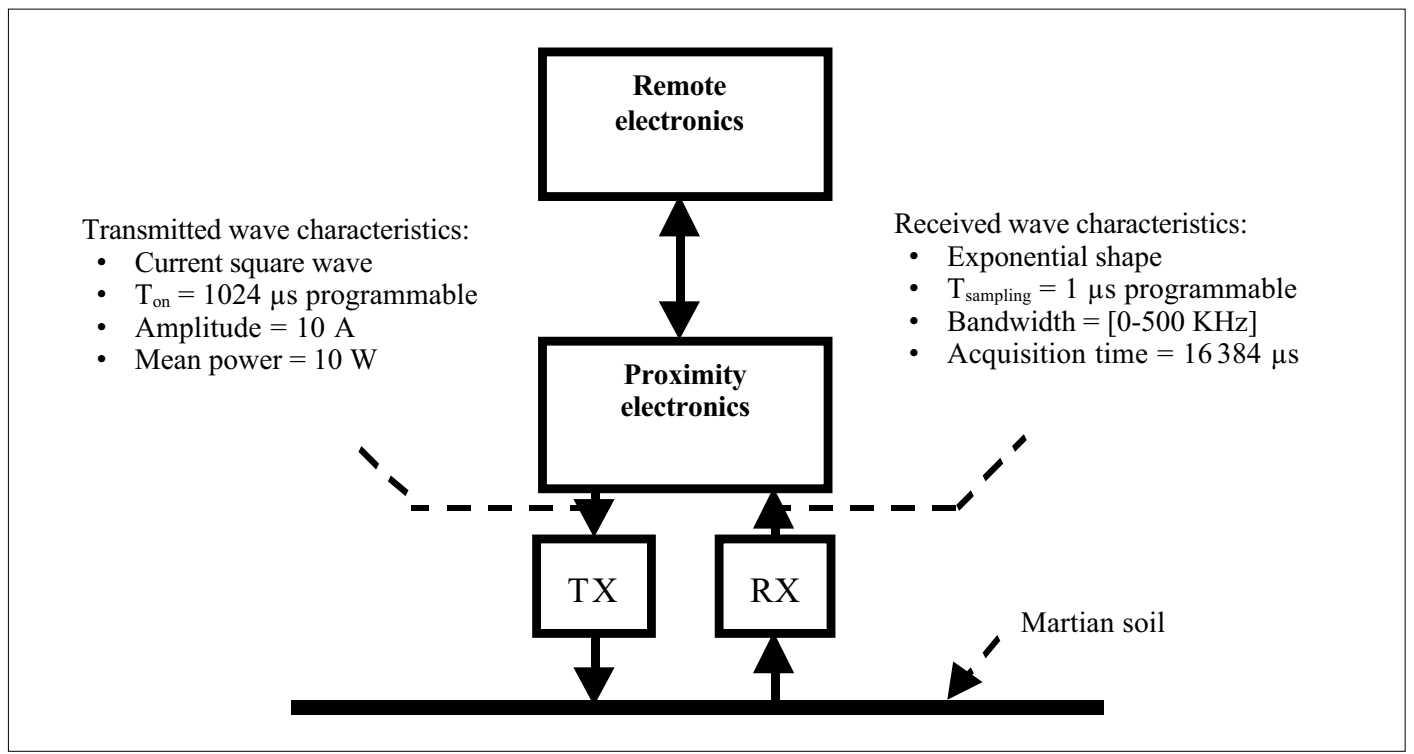

Fig. 2. Scheme of the TDEM system designed for DEEDRI. 
mitter and receiver) with a diameter of the order of $150 \mathrm{~mm}$. A logic scheme of MASTER is shown in fig. 2 .

It is mounted on the bottom face of the Drill box and does not interfere with the DEEDRI drilling and sampling activities. The coils directly face the soil (i.e. no conductive material shall be either interposed between the coils and the soil or allocated within the coils themselves). The total weight is $110 \mathrm{~g}$ for both coils. Coils are made up with copper conductors, with a section of 0.4 $\mathrm{mm}^{2}$. The coils should be contained in Teflon (or other material) coil holders. The coil holders have the double task of preserving the coils and of supporting them slightly spaced from the Lander fixing surface.

The control electronics is divided into two blocks: proximity electronics near the coils, and remote electronics which is part of the Main Electronics Unit Science boards.
The source signal or gate is a current square wave, the received signal is expected to have an exponential shape in the hypothesis of uniform laterally distributed electrical conductivity of the subsoil.

Data acquisition frequency is set to $1 \mathrm{MHz}$ with an acquisition time set to $16384 \mu \mathrm{s}$, but we foresee the operating option to scale it down to $500 \mathrm{KHz}$, thus extending the acquisition time to $32768 \mu$ s. Tele-commands from ground control are just a few scalar parameters such as a gate timing and sampling acquisition interval.

Mass and power restrictions are presented in tables I and II. They imply that the instrument is not presently configured as stand alone but shares some resources with the other subsystems of DEEDRI, i.e. data pre-processing unit, experiment management unit, power distribution unit (for operation and survival), and thermal control of electronics itself.

Table I. Mass budget of the TDEM system designed for DEEDRI Payload.

\begin{tabular}{llr}
\hline \multicolumn{1}{c}{ Subsystem } & \multicolumn{1}{c}{ Type } & Weight \\
\hline Transmitting coil & $\mathrm{Cu}$ - coil diameter $150 \mathrm{~mm}$, section $0.4 \mathrm{~mm}^{2}$ & $55 \mathrm{~g}$ \\
Receiving coil & $\mathrm{Cu}-$ coil diameter $150 \mathrm{~mm}$, section $0.4 \mathrm{~mm}^{2}$ & $55 \mathrm{~g}$ \\
Coils holder & Teflon & $130 \mathrm{~g}$ \\
Fixing bolts etc. & Stainless steel & $30 \mathrm{~g}$ \\
Proximity electronics box & $\mathrm{Al}$ & $70 \mathrm{~g}$ \\
Proximity electronics boards & & $90 \mathrm{~g}$ \\
Main electronics and harness & & $180 \mathrm{~g}$ \\
\hline Total weight of the system & & $610 \mathrm{~g}$ \\
\hline
\end{tabular}

Table II. Power budget of the TDEM system designed for DEEDRI Payload.

\begin{tabular}{lclr}
\hline \multicolumn{1}{c}{ Subsystem } & Status & \multicolumn{1}{c}{ Operation mode } & Power consumption \\
\hline Transmitting coil & & During pulse generation & $10 / 20 \mathrm{~W}$ \\
Keep-alive heaters $\left(^{*}\right)$ & Off & & $0.5 \mathrm{~W}$ \\
Proximity electronics & On & Stand-by & $0.8 \mathrm{~W}$ \\
Proximity electronics & On & Stand-by and pulse generation & $1.05 \mathrm{~W}$ \\
Main electronics (PC excluded) & On & Conversion and RAM management & $2.50 \mathrm{~W}$ \\
Peak of consumption & On & Measurement mode & $3.55 \mathrm{~W}$ \\
\hline
\end{tabular}

(*) The survival heaters switch off when proximity electronics reach the storage temperature range. 


\subsection{Signal acquisition and processing strategy}

In order to overcome limitations on data memory allocation and computation time, a measurement cycle was defined, that is the set of measurements and pre-processing procedures aimed at obtaining as much information as possible by reducing data exchange and memorization.

The operating procedure is based on a comparison of passive (no transmitted power) and active measured data.

The sampled signal is processed following two different criteria: the signals received after the transmission of the gate, called active measures, are averaged, Fast Fourier Transformed (FFT) and filtered according to a procedure based on the comparison with the signals received without a previous transmission, called passive measures. The scheme in fig. 3 represents the data processing block diagram.

The Modified Frequency Domain Kurtosis (MFDK) is applied to the vector of passive measures (Ottonello and Pagnan, 1994; Ottonello et al., 1994). The MFDK is a fourth order statistics operator that assigns a level of stationarity to each signal spectrum component and enhances unsteady components over stationary sinusoids embedded in noise. It is applied in order to remove local noise $n(t)$, which may be composed of slow time varying switching power supply sources $\left(n_{s}(t)\right)$, thermal noise $\left(n_{w}(t)\right)$, or EM interferences $\left(n_{\text {imp }}(t)\right)$. It is defined as the ratio between the fourth order moment of the FFT of the $i$-acquisition of the noise $n(t)\left(N_{i}(f)\right)$, with respect to the square of the second order moment of the FFT of the same signal, as follows:

$$
\operatorname{MFDK}(f)=\frac{\frac{1}{Q} \sum_{i=1}^{Q}\left|N_{i}(f)\right|^{4}}{\left[\frac{1}{Q} \sum_{i=1}^{Q}\left|N_{i}(f)\right|^{2}\right]^{2}} .
$$

This operator requires one FFT, two vectors multiplications and four vector sums for computation, but is very powerful in enhancing drifting components over stationary sinusoids. Theoretical results are interesting for detection of randomly occurring signals, when methods based on power spectrum may fail to accomplish this task.

This function is used to set, in an adaptive way, the digital filter $H(f)$. After this step, the useful signal is compressed maintaining only

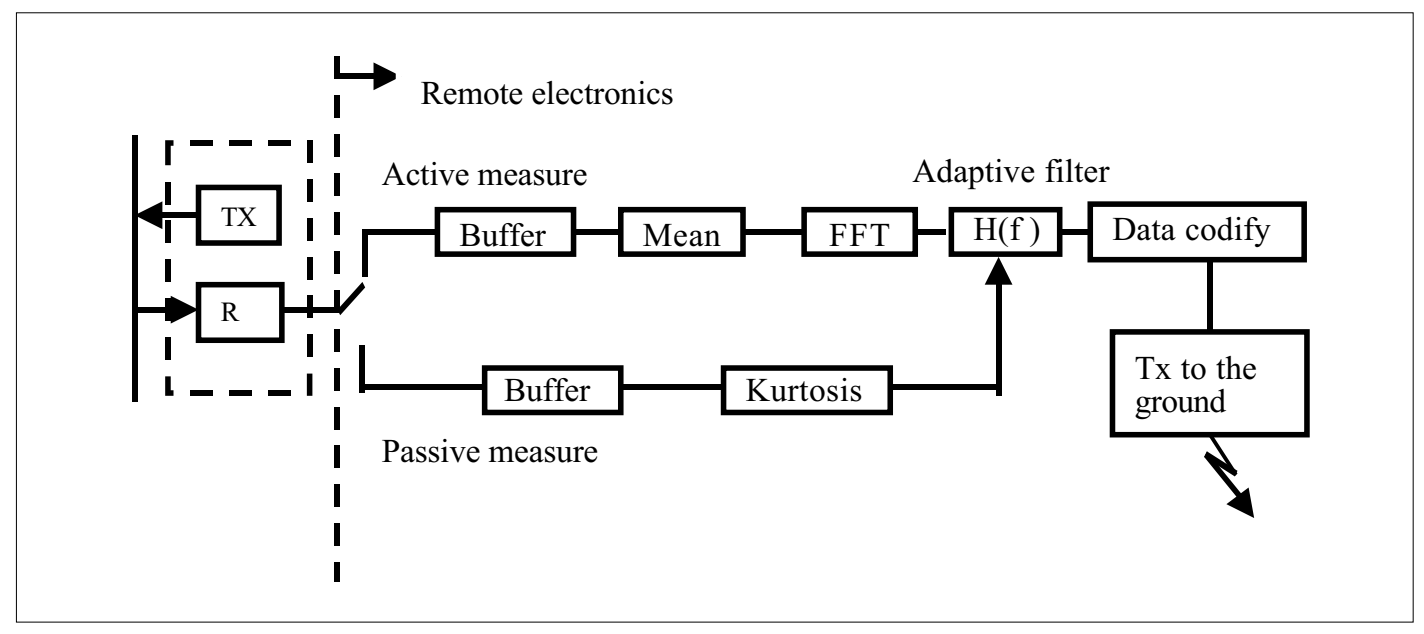

Fig. 3. Data processing block diagram. 
the information on the most powerful non stationary spectral lines. In detail, the operations timeline is as follows.

\subsubsection{Definition of a Single Measure (SM)}

A Single Measure implying a vector of length $N$ (default $N=16384$ ) is defined primarily as the set of measurements each one taken in sequence at small random delays (e.g., from 0 to $20 \mathrm{~s})$ with the drill mechanically stable and motors switched off.

All the data processing can be made in parallel or serialized, in order to minimize memory allocation and can run with lowest priority in background.

The sequence is:

1) Read a number $Q$ (default $Q=16$ ) of vectors in passive mode (without transmitting gate).

2) Upgrade the $M F D K$ state vector using the passive measures and the old state vectors taken from memory.

3) Read a number $L$ of vectors in active mode and perform the mean of $L$ active measures. The delay between measures can be random (e.g., 1 to $10 \mathrm{~s}$ ) and depends at least from the capacitor charging time in the transmission coil.

4) Perform the FFT of the mean.

5) Set to zero stationary frequency lines according to MFDK operator based processing (i.e. $H(f)$ filtering).

6) Sort in order of power magnitude the remaining spectral lines.

7) Take the first $M$ components (default $M=1024$ ) obtaining a reduced compressed vector.

8) Send or stack it, adding routine information like date, time, drill position, external temperature.

\subsubsection{Martian Day Timeline (MDT)}

1) Take care of commands from Earth, such as sample interval and programmable gate length.

2) If sample interval (default $=1 \mu \mathrm{s}$ ) or gate length has changed reset $M F D K$ state vector to zero.

3) If the drill changed position execute a Position Measure procedure, by sending to ground a new vector of passive measures and boundary information.

4) Distribute regularly or randomly over a Martian day a number $K$ of Single Measure (default $K=8$ ). If this process became unrealizable because of frequent drill activation, the $K$ Single Measures can be taken consecutively.

\subsection{Memory allocation and computation time}

By assigning the following default values to:

- data vector length: $N=16384$;

- number of repeated measurements in one SM: $Q=16$ (passive mode) and $L=16$ (active mode);

- reduced data vector length: $M=1024$ complex;

- number of SMs per Martian day: $K=4$;

- floating point of 5 bytes (40 bits);

- sampling time $T_{\text {sampling }}=1 \mu \mathrm{s}$, we obtain for one entire MDT: total acquisition time $\cong 2 \mathrm{~s} ;$ total computation FLOPs $=67.2$ MegaFlops; total bytes to Telemetry $=48$ Kbytes.

In order to reduce the number of data to be sent to Telemetry Data Subsystem, we may use, for example, $K=2$ and $Q=64$, obtaining 24 Kbytes per Martian day. This working configuration, although representing a minimal scenario for MASTER, covers the experiment's scientific validity.

\subsection{Simulation results}

A set of simulations were performed to validate the proposed data acquisition and compression strategy and to verify the performances of reconstruction algorithm versus compression parameters (i.e. $M$ value).

At first, we assumed that the Martian subsoil electrical conductivity is uniform laterally distributed. Hence, the subsoil conductivity is free to change layer by layer in depth, but it remains constant in the same layer (see fig. 4) (Hohmann, 1975; McNeill, 1984; Keller, 1994).

The relation between the subsoil electrical conductivity, the magnetic permeability and 


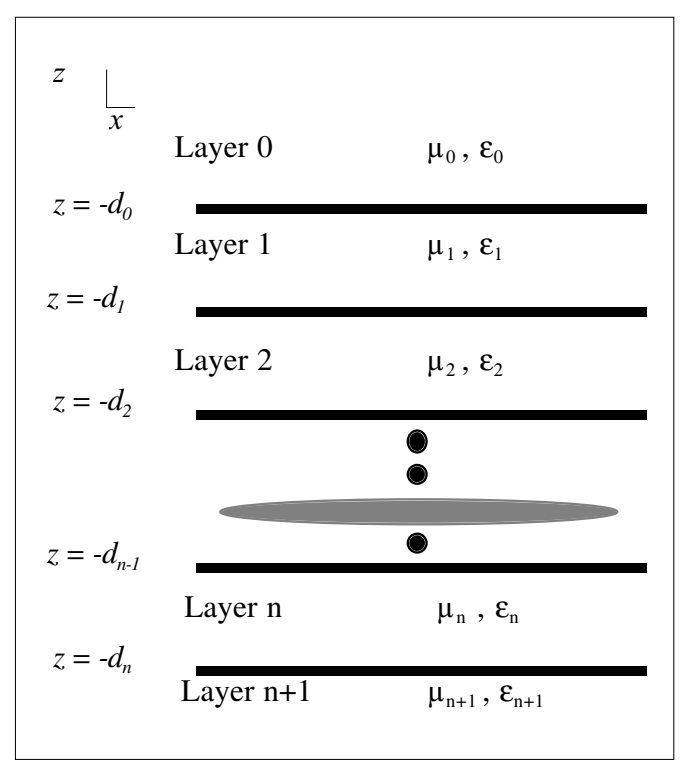

Fig. 4. Subsurface modelling.

the frequency of the received wave is the «skin depth» (Nabighian, 1994), defined as follows:

$$
\text { Skin depth } \delta \cong(\omega \mu \sigma)^{1 / 2}
$$

This well known relation allows geophysical interpretation of data, by correlating the information contained in the frequency spectrum of the returned signal with electrical conductivity.

In the simulation, the received signal $r(t)$ is modelled as the sum of two signals, as follows:

$$
r(t)=s(t)+n(t)
$$

where $s(t)=\Sigma_{i} a_{i} \cdot \exp \left(b_{i} t\right)$ represents the original signal and $n(t)$ is the noise, which can be modelled as the sum of three contributes:

$$
n(t)=n_{w}(t)+n_{s}(t)+n_{\text {imp }}(t)
$$

where $n_{w}(t)$ represents thermal noise (additive white Gaussian noise, i.e. AWGN), $n_{s}(t)$ is the noise derived by the lander on-board systems (sum of persistent sinusoids) and $n_{\text {imp }}(t)$ takes into account the impulsive components due to occasional EM interferences.

In order to test the performances of data processing and to evaluate its limitations, in the simulation tests different working scenarios were considered, changing the values of the operation timeline (i.e. $N, L, Q, M$ ) and the characteristics of $s(t)$ (e.g., amplitude, frequency, number of persistent sinusoids being within the noise bandwidth) and $n(t)$ (e.g., impulsiveness).

For each test, we compared the power spectrum of original signal $s(t)$ with respect to the power spectrum of the raw acquired signal $r(t)$ and of the reconstructed signal, after MFDK filtering and compression. In fig. 5, on the left the received raw signal is shown, while on the right, we can see the same signal after the processing. For comparison, the original signal $s(t)$ is reported in the small window.

The proposed algorithm proved efficient since:

- It is able to remove the periodic signals from the received signal power spectrum.

- It can attenuate the contribution of impulsive and occasional signals to noise.

- It can be executed in real time.

- It allows the maximum timeline flexibility.

The described data processing presents, however, some limitations: if parameters go over a range of values, the results could differ from the expected ones. In detail, the minimum number of passive acquisitions is $Q=16$, the maximum time interval of an impulsive signal is $500 \mu \mathrm{s}$; the minimum number of spectral lines to be transmitted to ground without complete loss of information is $M=128$.

\section{Conclusions}

The paper presented the purposes of a new project, TESEO, which, collecting the results of the feasibility study of MASTER system, intends to answer the growing interest in EM sounding systems to be installed aboard carriers (landers, rovers, drills) to be delivered on Solar System body surfaces.

The presentation mainly concerned the engineering and telecommunication problems 

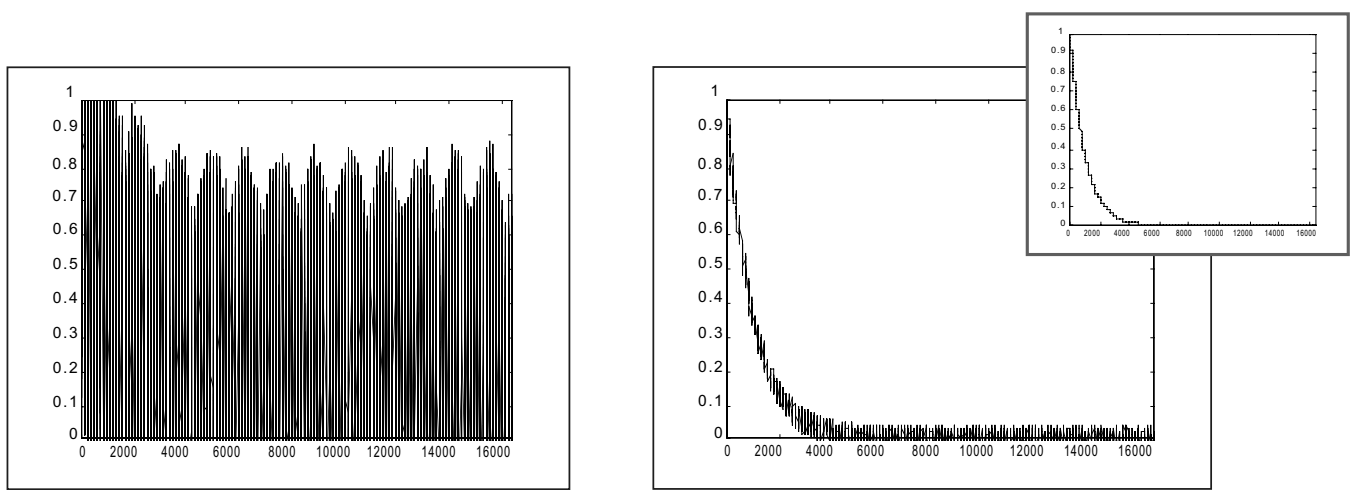

Fig. 5. Simulation results. On the left side: the received signal $r(t)$. For the test the noise was modelled as the sum of two sinusoids with a maximum amplitude equal to 0.6, an AWGN with a maximum amplitude equal to 0.2 and a spike with a maximum amplitude equal to 0.3 and a duration of $500 \mu$ s. On the right side: the processed signal obtained after filtering and compressing procedures; in the small window: the original signal $s(t)$.

related to data acquisition and processing, not the geophysical interpretation aspects, which are far from the expertise of the authors.

The TDEM technique can be successfully applied for performing non intrusive in-situ geophysical measures on planetary bodies, although several technological problems still need to be addressed. For example for the MASTER system, the following problems need to be assessed:

- Increasing transmitting coil current by using supercapacitor recharging systems.

- Implementing a receiving coil based on SQUID (Superconducting Quantum Interference Device) technology.

- Optimizing data compression and transmission algorithms.

TDEM instruments are strongly recommended as reference ground truth for the remote sensing missions where data is acquired by sounding radars from satellites. TDEM data will serve as a comparison reference for other scientists willing to explore the subsurface properties, employing different scientific investigation techniques, thus inspiring a variety of new insights into geologic history and up-to-date structure of planetary bodies. Applications will mainly concern the 2005-2007 Mars Sample Return Missions but may be extended to space mis- sions on other bodies of the Solar System: Moon, Mercury, Venus, Titan.

Solutions adopted for space application may also have a significant impact on ground surveying, as concerns for example the following aspects:

- Lightweight and portable systems may improve applications in irregular and inaccessible terrains and/or may speed the execution of measures over large areas;

- Reduced equipment dimensions are also required for applications concerning natural hazards prevention (e.g., volcanic eruptions) and cultural heritage inspection and monitoring.

- Solutions for reducing measure allocation resources (e.g., by using suitable compression algorithms) and implementing more efficient reconstruction and processing algorithms for noisy or corrupted data may be transferred from space to ground applications.

\section{Acknowledgements}

The authors wish to thank the other partners involved in the TESEO proposal preparation: Prof. Domenico Patella, Department of Physical Sciences, University «Federico II» of Naples, 
Prof. Tiziano Cantalupi, Osservatorio Geofisico «R. Bendandi», Faenza; Dr. Goro Komatsu, International Research School of Planetary Sciences, Università d'Annunzio, Pescara. Particular thanks to Prof. Giorgio Tacconi for his valuable suggestions to the research.

\section{REFERENCES}

HohmanN, G.W. (1975): Three Dimensional Induced Polarization and Electromagnetic Modelling, Geophysics, 40, 309-324.

Keller, G.V. (1994): The Geoelectrical Method in Geophysical Exploration (Elsevier).

MCNEILL, J.D. (1984): Approximate calculations of the transient electromagnetic response from buried conductors in a conductive half-space, Geophysics, 49 (7), 918-924.

NABIGHIAN, M.N. (Editor) (1984): Special issue on time domain electromagnetic methods of exploration, Geophysics, 49 (7), 849-1029.

NABIGHIAN, M.N. (Editor) (1993): Electromagnetic methods in applied geophysics, Soc. Explor. Geophys., 1-2.

OTTONELlO, C. and S. PAGNAN (1994): Modified frequency domain kurtosis for signal processing, Electron. Lett., 30 (14), 1117-1118.

OTTONELlO C., S. PAGNAN and G. TACCONI (1994): Filtering of randomly occurring signals by kurtosis in frequency domain, in 12th International Conference on Pattern Recognition, Jerusalem, Israel, October 9-13, 1994, vol. 3, 131-133.

TACCONI, G., S. PAgnan, L. Minna, R. MARCIAlis, G. BuCCI and C. OTTONELLO (2000): Mars exploration by TDEM based measurement system, in Proceedings of PIERS, July 5-14, 2000, Cambridge, Ma, U.S.A., p. 1067. 